\title{
Role of caregiver-reported outcomes in identification of children with prenatal alcohol exposure during the first year of life
}

\author{
Ludmila N Bakhireva ${ }^{1}$, Jean Lowe ${ }^{2}$, Laura M Garrison ${ }^{1}$, Sandra Cano ${ }^{1}$, Yuridia Leyva ${ }^{3}$, Fares Qeadan ${ }^{3}$ and Julia M Stephen ${ }^{4}$
}

BACKGROUND: Earlier identification of children with prenatal alcohol exposure (PAE) remains a challenge. The objective of this study was to identify neurobehavioral (NB) outcomes associated with PAE in infants.

METHODS: This manuscript evaluates NB outcomes at $6.33 \pm 1.12$ months of age in 93 infants (39 PAE and 54 NoPAE) recruited prospectively into the ENRICH cohort. PAE was assessed by prospective repeated TLFB interviews and a panel of ethanol biomarkers. NB outcomes were evaluated by the Bayley Scales of Infant Development (BSID-III), Parenting Stress Index (PSI), Infant Behavior Questionnaire (IBQ-R), and Infant Sensory Profile (ISP).

RESULTS: Mean maternal age at enrollment was $28.18 \pm 5.75$, and $64.52 \%$ were Hispanic/Latina. Across three TLFB calendars, absolute alcohol per day in the PAE group was $0.44 \pm 0.72$, corresponding to low-moderate alcohol consumption. While no association was observed between PAE and BSID-III $\left(P^{\prime} S>0.05\right), P A E$ was associated with higher scores on the PSI difficult child scale $(\hat{\beta}=13.9 ; \quad P=0.015)$, total stress $(\hat{\beta}=13.9 ; \quad P=0.010)$, and IBQ negative affect $(\hat{\beta}=8.60$; $P=0.008)$ measures after adjustment for covariates.

CONCLUSIONS: Caregiver-reported assessments may provide a currently unrecognized opportunity to identify behavioral deficits, point to early interventions, and should be included in clinical assessments of infants at-risk for fetal alcohol spectrum disorder.

A n estimated $18 \%$ of US pregnancies are affected by prenatal alcohol exposure (PAE) (1). PAE can result in the development of lifelong disabilities, collectively termed fetal alcohol spectrum disorders (FASDs). Recent reports indicate that FASD prevalence is much higher than previously thought and might affect as many as $2-5 \%$ of school-aged children (2). Although FASD is associated with a wide range of adverse physical and neurological impairments, many individuals on the spectrum have severe neurobehavioral (NB) deficits without characteristic physical features, potentially hindering their early and accurate diagnosis and access to appropriate interventions. The diagnosis of FASD commonly is not reached until an affected child is well into school-age years. A recent study demonstrated that as many as $86.5 \%$ of school-aged children with FASD are undiagnosed or misdiagnosed (3). Such challenges in early and accurate identification can be attributed to lack of training and awareness among medical and education professionals, challenges related to differential diagnosis, co-occurrence of FASD with other neurodevelopmental disorders (4), and the dearth of validated behavioral screening and diagnostic tools, particularly for use in infants and toddlers. Without early and effective interventions, these NB deficits can lead to secondary adverse outcomes, such as poor academic performance and sociability, unemployment, substance abuse, homelessness, and incarceration (5).

The neuropsychological profile of persons with FASD is marked by profound variability, potentially affecting a number of different domains including executive function, learning and memory, language, visual-spatial skills, motor function, attention and activity, academic performance, and behavior (6). It has been widely agreed that a variety of standardized measures be incorporated in clinical evaluations for FASD; thus, numerous NB assessments have been studied for screening and diagnostic purposes. These assessments have been heavily concentrated in children of preschool-age or older, with findings from preschool-age samples suggesting possible feasibility in extending $\mathrm{NB}$ assessment to even younger populations $(7,8)$. Molteno et al. (9) found that diminished competence in symbolic play at 13 months and emotional withdrawal at 6.5 months of age (10) were significantly altered in PAE infants from a South African cohort; findings at 6.5 months were significantly predictive of low IQ at a 9-year follow-up. In another South African cohort, Davies et al. (11) found that infants and toddlers with full and partial fetal alcohol syndrome (FAS, pFAS) performed worse than those without FAS or pFAS on all sub-scales and on the total development quotient of the Griffith's Mental

\footnotetext{
${ }^{1}$ Pharmacy Practice and Administrative Sciences, College of Pharmacy, University of New Mexico, Albuquerque, New Mexico; ${ }^{2}$ Department of Pediatrics, University of New Mexico, Albuquerque, New Mexico; ${ }^{3}$ Division of Epidemiology, Biostatistics, and Preventive Medicine, Department of Internal Medicine, University of New Mexico, Albuquerque, New Mexico; ${ }^{4}$ The Mind Research Network and Lovelace Biomedical and Environmental Research Institute, Albuquerque, New Mexico. Correspondence: Ludmila N. Bakhireva (lbakhireva@salud.unm.edu) 


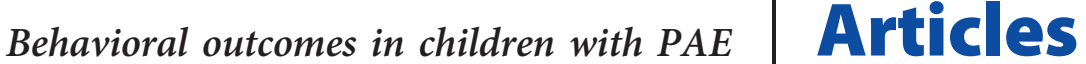

Development Scale. A prospective cohort study in Ukraine found that PAE was associated with lower Mental Development Index on the Bayley Scales of Infant Development-II (BSID-II) (12) and alterations in the cardiac orienting response at 6 and 12 months of age (13). In an earlier study at the University of New Mexico, we demonstrated altered emotional regulation and stress reactivity during the Still-Face paradigm in 5-7-month-old infants affected by PAE (14).

Although the published results are limited, they support the hypothesis that NB deficits related to PAE may be detectable in children younger than 2 years of age. Given that higherorder NB domains, such as executive functioning, only begin to emerge around 18 months of age, early identification of children affected by PAE remains one of the biggest challenges in the field. The objective of this prospective cohort study was to identify $\mathrm{NB}$ indices associated with moderate PAE in 5-8-month-old infants. We hypothesized that impairments in self-regulation and sensory processing domains will be more sensitive measures of PAE than NB indices obtained on standardized global assessment tests, such as BSID. The long-term goal of our program is to refine analytical procedures with greater utility for the identification of children with PAE at an earlier stage in development.

\section{METHODS}

\section{Study Design and Population}

The data for this study were obtained from the prospective birth cohort, Ethanol, Neurodevelopment, Infant and Child Health (ENRICH), at the University of New Mexico (UNM), the methodology of which has been described elsewhere (15). Briefly, this study consists of four visits: enrollment and baseline data collection obtained during pregnancy (Visit 1 [V1]), evaluation during the hospital stay after labor and delivery (Visit 2 [V2]), a 5-8month assessment of the infant (Visit 3 [V3]), and a 20-24-month follow-up assessment (Visit 4 [V4]). All study activities were reviewed and approved by the UNM Health Sciences Center Human Research Protections Program, and all participants provided written informed consent. The study began in 2013, and as of September 2017, 93 maternal-infant dyads had completed the first three visits and were included in the analyses presented in this manuscript. The overall retention rate was $72.1 \%$.

Participants were recruited from UNM hospital-affiliated prenatal care clinics, including a specialty prenatal care program, called Milagro, which exclusively treats pregnant and postpartum women with substance use disorders. Participants were recruited into four study groups: (1) PAE; (2) medication-assisted therapy (MAT) with opioid agonists for opioid use disorder; (3) PAE+MAT; and (4) unexposed/healthy controls (HC). Individuals with MAT were included to better match pre- and post-natal environmental factors and socio-economic status (SES) across groups. Among subjects on MAT, 17 were on methadone, 22 on buprenorphine, and 2 had exposure to both regiments. To examine the primary hypothesis regarding the effects of PAE on infant development, these four groups were combined into two: PAE (PAE with or without MAT) and No-PAE (MAT and HC).

Inclusion criteria for all study groups include: (1) a singleton pregnancy, (2) enrolled between 12 and 35 gestational weeks, (3) delivered at UNM hospital, (4) planning to reside in New Mexico for 2 years to complete all study visits, and (5) ability to provide written consent in English. Exclusion criteria include: (1) fetal diagnosis of a major structural abnormality and (2) more than occasional $(>1$ urine drug test or more than monthly) use of cocaine, methamphetamines, or MDMA during the first trimester and any use of these substances in the second or third trimesters as determined by either self-report, positive urine drug screen, or medical records review. Study participants in the PAE, MAT, and PAE+MAT groups were not disqualified due to concurrent use of nicotine or non-stimulant substances of abuse (e.g., marijuana). HC participants were those who reported no alcohol use after the last menstrual period (LMP), tested negative on all biomarkers (described below), and were abstainers from tobacco and illicit drug use. During recruitment, the goal was to frequency match groups on SES, and recruitment was adjusted throughout to best match groups.

\section{Assessment of Prenatal Alcohol Exposure}

PAE was assessed by repeated prospective Timeline Follow-Back (TLFB) (16) 30-day calendar interviews and a comprehensive battery of ethanol biomarkers. At baseline (prenatal/V1), TLFB was administered for the periconceptional period (2 weeks before and 2 weeks after the LMP; TLFB 1 ), and the 30 days leading up to enrollment $\left(\mathrm{TLFB}_{2}\right)$. At V2 (delivery), TLFB was administered for the 30 days preceding the visit $\left(\mathrm{TLFB}_{3}\right)$. Moreover, alcohol use during "special occasions" outside of these time periods was also recorded. In addition to self-report, this study involved assessment of a battery of ethanol biomarkers. At V1, gamma-glutamyl transpeptidase (GGT), carbohydrate-deficient transferrin (CDT), and phosphatidylethanol (PEth) were analyzed from maternal blood specimens. At V2, GGT, CDT, and PEth analyses were repeated and supplemented with maternal urine ethylgucuronide (uEtG) and ethylsulfate (uEtS), and PEth in a newborn dry blood spot card (PEth-DBS). Collection and laboratory processes pertaining to these biomarkers have been described in detail elsewhere $(15,17)$.

Participants who report more than minimal-risk alcohol use $(>13$ standard drink units (SDU)/month) or $\geq 2$ binge episodes in the periconceptional period and continue to use alcohol during pregnancy (as evident by alcohol use on $\mathrm{TLFB}_{2}$ or $\mathrm{TLFB}_{3}$ or positivity on $\geq 1$ ethanol biomarker) met criteria for the PAE groups. Initial categorization was based on the $\mathrm{TLFB}_{1}$ since hazardous drinking in the periconceptional period is highly predictive of alcohol use later in pregnancy, is reported more openly (16), and is a good predictor of infant neurodevelopmental outcomes later in life (12). Confirmation by $\mathrm{TLFB}_{2}, \mathrm{TLFB}_{3}$, and ethanol biomarkers was done to ensure PAE exposure after pregnancy recognition. These criteria, incorporating "more than minimal" risk alcohol exposure levels, are consistent with the definition of PAE in the DSM-5 proposed classification for neurodevelopmental disorder associated with PAE (ND-PAE) (18). Subjects were classified into non-PAE groups if they (a) reported no more than "light" drinking ( $\leq 2$ drinks/ week) in the periconceptional period, (b) reported abstinence from alcohol use during pregnancy (all three TLFB calendars and special occasions), and (c) tested negative on all ethanol biomarkers. Subjects who initially were classified into the control groups but later tested positive for ethanol biomarkers were disqualified and did not continue to V3.

\section{Assessment of NB Outcomes}

At V3 (5-8-month infant assessment), NB outcomes were assessed using a battery of validated measures. This included the administration of gross motor, fine motor, cognitive, and language scales of the BSID-III by a pediatric developmental diagnostician (J.L.), who was blinded to the exposure status. In addition, a number of validated caregiver questionnaires were administered to assess the following: (1) quality of parent-child interactions and parenting stress (Parenting Stress Index-Short Form [PSI-SF]); (2) infant temperament (Infant Behavior Questionnaire-Revised [IBQ-R]); and (3) infant sensory processing and reactivity (Infant/Toddler Sensory Profile [ISP]). Specific domains evaluated by each instrument are summarized in Table 1. All assessments were scored by trained members of the research team who were blinded to participants' exposure status. At the baseline visit, patients were encouraged to discuss alcohol use with their healthcare providers; the majority already were receiving comprehensive care through the Milagro 


\section{Articles Bakhireva et al.}

Table 1. Assessment battery of infant outcomes

\begin{tabular}{|c|c|c|}
\hline Domain & Instrument-subscale & Subscale description \\
\hline \multirow[t]{4}{*}{ Infant development } & BSID-III & \\
\hline & - Cognitive & Novelty interest/attention, problem-solving, pretend play \\
\hline & - Language & Receptive and expressive communication \\
\hline & - Motor & Fine and gross motor skills \\
\hline \multirow[t]{6}{*}{ Infant temperament } & Infant Behavior Questionnaire-R & \\
\hline & - Surgency & Externalizing behaviors \\
\hline & - Negative affect & Fearfulness, emotional distress \\
\hline & - Effortful control & Self-regulation (inhibitory control, attentional regulation, activational control) \\
\hline & Parenting Stress Index-Short & Disruptive or destructive behaviors \\
\hline & - Difficult child & likely to contribute to a parent's stress \\
\hline \multirow[t]{3}{*}{ Parental stress } & Parenting Stress Index-Short & \\
\hline & $\begin{array}{l}\text { - Parent-child dysfunctional } \\
\text { interaction }\end{array}$ & Quality of interactions, parent's relationship expectations and satisfaction \\
\hline & - Parental distress & $\begin{array}{l}\text { Parent's personal adjustment to parenting, parent's relationship with child's other } \\
\text { parent (e.g., spouse), other life stressors }\end{array}$ \\
\hline \multirow[t]{4}{*}{ Infant sensory processing } & Infant/Toddler Sensory Profile & \\
\hline & - Seeking & Interest in and pleasure with stimuli \\
\hline & - Sensitivity & Ability to notice sensations \\
\hline & - Avoiding & Effort to control or minimize sensations \\
\hline
\end{tabular}

program. At the completion of $\mathrm{V} 3$, all participants received a summary of their infant's neurodevelopmental assessment; necessary referrals to early intervention programs were made.

\section{Assessment of Covariates}

Prenatal exposure to all major substances of abuse (nicotine, cocaine, methamphetamines, 3,4-methylenedioxymethamphetamine (MDMA or "ecstasy"), nonmedical use of opioid analgesics, heroin, marijuana/ cannabinoids, benzodiazepines, and barbiturates) was assessed by repeated maternal report, a study-specific 7-panel urine drug test analyzed by Tricore Reference Laboratories (Albuquerque, New Mexico), and abstraction of urine drug screen results from electronic medical records (EMR) (15). Maternal demographic, socioeconomic, medical, and reproductive health characteristics were collected at V1, and birth outcomes were abstracted from participants' EMR. Maternal Perceived Stress Scale (PSS) (19) was administered at V2 to capture maternal perceived stress at the end of pregnancy (30 days before delivery). Additionally, postnatal risk factors were assessed at V3, including maternal depressive symptoms (Beck Depression Inventory [BDI]), household income, and utilization of social services and participation in early intervention programs.

\section{Statistical Analyses}

Power calculations were done a priori for the BSID-III assuming a population mean of 100 with $S D=15$. With sample sizes of 39 and 54 , we had $93.4 \%$ power to detect a 10 -point difference between the study groups. This difference translates to Cohen's $d$ effect size of 0.67 , where $d=0.2$ is considered a "small" effect size, 0.5 represents a "medium" effect size, and 0.8 a "large" effect size. The sample size of 93 subjects is sufficient to adjust for 9 covariates in a multiple linear regression setting while maintaining at least 10 subject per variable (SPV) which in turns assures no overfitting.

Numerical and graphical summaries were computed for all measurements obtained from the maternal prenatal and early postpartum interviews and results of laboratory analyses. T-tests and Chi-square tests were used to compare means and proportions of continuous and categorical variables, respectively, between the study groups. For variables not normally distributed (e.g., BDI), the Wilcoxon rank-sum test for differences in medians was used. Linear regression was conducted to investigate the effect of PAE on continuously distributed infant outcomes (i.e., BSID, PSI, and IBQ scores). Ordinal sensory profile variables (less than others, typical performance, more than others) were dichotomized into atypical performance (less/more than others) vs. typical performance. The binary logistic regression was conducted to investigate the effect of PAE before and after adjustment of covariates on these outcomes. For multivariable analyses, a list of covariates was selected a priori based on known risk and resilience factors affecting infant neurodevelopmental outcomes (i.e., infant sex, birth weight, single vs. two-parent household, BDI, maternal education, maternal age) and co-exposures that were differentially distributed between the study groups (i.e., MAT, marijuana, tobacco use); all are considered to be biologically meaningful. Of note, while the PAE group had a higher prevalence of preterm delivery, gestational age at delivery was not included as a covariate due to its strong collinearity with birth weight (tolerance $\approx 0.44, \mathrm{VIF}=2.3$, Pearson correlation coefficient $0.68 ; P<0.0001)$ and because infant age at V3 assessment was already adjusted for prematurity. A sensitivity analysis was conducted to identify the best subset of covariates using a stepwise backwards selection procedure. The results of the best subset models were similar to those of the full models, thus results were presented after adjustment for a full set of covariates as a more conservative approach. In addition to the reported $P$-values, measures of effect size (partial eta squared) were provided to assist in better interpretation of the finding where $\eta^{2}=0.01, \eta^{2}=0.06$, and $\eta^{2}$ $=0.14$ indicate small, medium, and large effect sizes, respectively.

\section{RESULTS}

The mean maternal age at enrollment was $28.18 \pm 5.75$ years, and the majority of subjects were recruited during the second trimester (gestational age at enrollment: $24.46 \pm 7.36$ weeks). There were no significant differences in socio-demographic and medical characteristics (age, ethnicity, marital/co-habituating 


\section{Behavioral outcomes in children with PAE Articles}

status, education, gravidity, SES, Beck Depression Inventory, and annual family income) between the study groups except race (Table 2). While the study recruited 64.5\% Hispanic Latina women who were similarly distributed between two study groups, the PAE group included a higher proportion of other minorities compared to the No-PAE group $(P=0.010)$. Additionally, a higher proportion of subjects in the PAE group participated in an early intervention program (58.3\% vs. $35.1 \%$ ); however, differences did not reach statistical significance $(P=0.087)$. Subjects in the PAE group also had a higher mean PSS score compared to No-PAE $(13.4 \pm 5.7$ vs. $10.0 \pm 4.9$, respectively; $P=0.003$ ). With respect to pregnancy outcomes, the PAE group had a higher prevalence of preterm delivery (25.6\% vs. $5.6 \%)$ and lower infant birth weight $(2,881 \pm 699 \mathrm{~g}$ vs. $3,209 \pm 582 \mathrm{~g}$; both $P$ 's $<0.05)$. Infant age at V3 assessment was similar in both groups.

In accordance with the eligibility criteria, the No-PAE group reported minimal alcohol use in the periconceptional period $(0.003 \pm 0.009)$, no alcohol use beyond the periconceptional period, and tested negative on all ethanol biomarkers at V1 and V2 (Table 3). The PAE group reported heavy/risky alcohol use in the periconceptional period $(1.27 \pm 2.16 \mathrm{AA} /$ day, equivalent to approximately 18 standard drinks per week) with, on average, $7.24 \pm 8.97$ binge drinking episodes per reported 30-day calendar. The alcohol consumption decreased later in pregnancy, with the reported AA/day at $\mathrm{V} 1$ being only $0.05 \pm 0.25$ (equivalent to 0.7 standard drinks/ week). Across three TLFB calendars, AA/day in the PAE group was $0.44 \pm 0.72$ (equivalent to approximately 6 drinks/ week), which is considered moderate drinking (20). Notably, $53.9 \%$ of subjects in the PAE group tested positive for at least one ethanol biomarker at either V1 or V2. Consistent with the eligibility criteria, $56.4 \%$ and $35.2 \%$ of patients in the PAE and No-PAE groups, respectively, were receiving MAT $(P=0.057)$. Co-exposure with other opioids was also prevalent but similar in both groups $(P=0.105)$. Marijuana use (35.9\% vs. $11.1 \%)$ and tobacco use (53.9\% vs. $27.8 \%)$ were more prevalent in the PAE group (both $P^{\prime} s<0.05$ ).

Infant developmental and behavioral outcomes by study group are presented in Table 4. No differences in the BSID-III cognitive, language, or motor scores were observed between PAE and No-PAE subjects ( $P$ 's $>0.05)$. On the Parenting Stress Index (PSI) and Infant Behavior Questionnaire (IBQ), children in the PAE group had higher scores for parent-child dysfunctional interaction $(P=0.019)$, difficult child $(P=0.011)$, total stress score $(P=0.011)$, and infant negative affect $(P=0.011)$ compared to the No-PAE group. No differences were observed between the groups for the Sensory Profile indices $(P>0.05)$.

Results of multivariable analyses demonstrated that PAE was associated with higher scores on the PSI difficult child $(\hat{\beta}=13.9 ; P=0.015)$, total stress $(\hat{\beta}=13.9 ; P=0.010)$, and IBQ negative affect $(\hat{\beta}=8.60 ; \quad P=0.008)$ scales after
Table 2. Demographic and medical characteristics of participants $(N=93)$

\begin{tabular}{|c|c|c|c|}
\hline Patient characteristics & $\begin{array}{l}\text { PAE } \\
(n=39)\end{array}$ & $\begin{array}{l}\text { No-PAE } \\
(n=54)\end{array}$ & $P$ \\
\hline & Mean \pm SD & Mean $\pm S D$ & \\
\hline Maternal age at enrollment (years) & $28.95 \pm 5.88$ & $27.63 \pm 5.64$ & 0.277 \\
\hline Gestational age at enrollment (weeks) & $25.69 \pm 6.99$ & $23.58 \pm 7.56$ & 0.174 \\
\hline Gestational age at delivery (weeks) & $38.25 \pm 2.85$ & $39.09 \pm 1.41$ & 0.096 \\
\hline Infant birth weight (grams) & $2881 \pm 699$ & $3209 \pm 582$ & 0.016 \\
\hline Infant age at V3 assessment (months) ${ }^{\mathrm{a}}$ & $6.49 \pm 1.19$ & $6.22 \pm 1.06$ & 0.261 \\
\hline Total SES score ${ }^{\mathrm{b}}$ & $30.8 \pm 13.2$ & $31.1 \pm 12.8$ & 0.934 \\
\hline Maternal BDI score $(\text { Median } \pm I Q R)^{c}$ & $7.0 \pm 7.0$ & $5.5 \pm 8.0$ & 0.384 \\
\hline \multirow[t]{2}{*}{ Maternal PSS score } & $13.4 \pm 5.7$ & $10.0 \pm 4.9$ & 0.003 \\
\hline & $n(\%)$ & $n(\%)$ & \\
\hline Preterm delivery & $10(25.6)$ & $3(5.6)$ & 0.013 \\
\hline Ethnicity & & & 0.385 \\
\hline Hispanic/Latina & $23(59.0)$ & $37(68.5)$ & \\
\hline Non-Hispanic/Latina & $16(41.0)$ & $17(31.5)$ & \\
\hline Race & & & 0.010 \\
\hline White & $30(76.9)$ & $52(96.3)$ & \\
\hline African American & $2(5.1)$ & $0(0.0)$ & \\
\hline American Indian & $6(15.4)$ & $1(1.9)$ & \\
\hline Other & $1(2.6)$ & $1(1.9)$ & \\
\hline Marital/cohabiting status: & & & 1.000 \\
\hline Single/separated/divorced & $17(43.6)$ & $23(42.6)$ & \\
\hline Married/cohabitating & $22(56.4)$ & $31(57.4)$ & \\
\hline Education level: & & & 0.959 \\
\hline Less than high school & $12(30.8)$ & $15(27.8)$ & \\
\hline High school to some college & $20(51.3)$ & $30(55.6)$ & \\
\hline College/professional degree & $7(18.0)$ & $9(16.7)$ & \\
\hline Primigravida & & & 0.343 \\
\hline Yes & $8(20.5)$ & $17(31.5)$ & \\
\hline No & $31(79.5)$ & $37(68.5)$ & \\
\hline Infant's gender: & & & 0.403 \\
\hline Male & $17(43.6)$ & $29(53.7)$ & \\
\hline Female & $22(56.4)$ & $25(46.3)$ & \\
\hline $\mathrm{BDI}>13(\%)$ & $5(13.5)$ & $9(16.7)$ & 0.774 \\
\hline Family gross annual income at V3 (\%) & & & 0.695 \\
\hline Under $\$ 20,000$ & $15(38.5)$ & $20(37.7)$ & \\
\hline$\$ 20,000-39,999$ & $13(33.3)$ & $14(26.4)$ & \\
\hline$\$ 40,000$ or over & $11(28.2)$ & 19 (35.9) & \\
\hline $\begin{array}{l}\text { Participated in early intervention } \\
\text { program }\end{array}$ & $21(55.3)$ & 19 (35.9) & 0.087 \\
\hline
\end{tabular}

Sample size for specific variables might vary due to pairwise deletion of the missing data ; median and IQR is presented due to data not being normally distributed. BDI, Beck Depression Inventory; IQR, interquartile range; PSS, perceived stress score. ${ }^{a}$ Age at assessment was adjusted for prematurity.

${ }^{b}$ Barratt simplified measure of social status.

${ }^{c} P$-value reflects Wilcoxon test for difference in medians. 
Table 3. Alcohol and substance use patterns by study group $(N=93)$

\begin{tabular}{|c|c|c|c|}
\hline & PAE $(n=39)$ & No-PAE $(n=54)$ & $P$ \\
\hline \multicolumn{4}{|c|}{ ALCOHOL USE } \\
\hline \multicolumn{4}{|l|}{12 months prior to enrollment: } \\
\hline $\begin{array}{l}\text { AUDIT past } 12 \text { months: } \\
(\text { mean } \pm \text { SD) }\end{array}$ & $10.31 \pm 7.74$ & $0.57 \pm 0.74$ & $<0.001$ \\
\hline AUDIT $\geq 8: n(\%)$ & $21(53.9)$ & $0(0.0)$ & $<0.001$ \\
\hline \multicolumn{4}{|c|}{ Periconceptional period (1 month around LMP) } \\
\hline $\mathrm{AA} /$ day $($ mean $\pm \mathrm{SD})$ & $1.27 \pm 2.16$ & $0.0026 \pm 0.009$ & $<0.001$ \\
\hline $\mathrm{AA} /$ drinking day $($ mean $\pm \mathrm{SD})$ & $3.17 \pm 2.09$ & $0.86 \pm 0.42^{\mathrm{a}}$ & $<0.001$ \\
\hline $\begin{array}{l}\text { Number of binge episodes } \\
\text { (mean } \pm \text { SD) }\end{array}$ & $7.24 \pm 8.97$ & $0 \pm 0$ & $<0.001$ \\
\hline \multicolumn{4}{|l|}{ Alcohol use at V1: } \\
\hline $\mathrm{AA} /$ day $($ mean $\pm \mathrm{SD})$ & $0.05 \pm 0.25$ & $0 \pm 0$ & 0.237 \\
\hline $\mathrm{AA} /$ drinking day $($ mean $\pm \mathrm{SD})$ & $2.11 \pm 2.49$ & N/A & $\mathrm{N} / \mathrm{A}$ \\
\hline $\begin{array}{l}\text { Number of binge episodes } \\
\text { (mean } \pm \text { SD) }\end{array}$ & $0.21 \pm 1.13$ & $0 \pm 0$ & 0.263 \\
\hline \multicolumn{4}{|l|}{ Maternal alcohol biomarkers at V1: } \\
\hline GGT ( > 40 U/I): $n(\%)$ & $9(23.1)$ & $0(0.0)$ & $<0.001$ \\
\hline PEth ( $\geq 8$ ng/ml): $n$ (\%) & $4(10.3)$ & $0(0.0)$ & 0.028 \\
\hline$\% \mathrm{dCDT}>2: n(\%)$ & $2(5.1)$ & $0(0.0)$ & 0.173 \\
\hline \multicolumn{4}{|l|}{ Alcohol use at V2: } \\
\hline $\mathrm{AA} /$ day $($ mean $\pm \mathrm{SD})$ & $0.004 \pm 0.014$ & $0 \pm 0$ & 0.077 \\
\hline $\mathrm{AA} /$ drinking day $($ mean $\pm \mathrm{SD})$ & $0.97 \pm 0.90$ & N/A & $\mathrm{N} / \mathrm{A}$ \\
\hline $\begin{array}{l}\text { Number of binge episodes } \\
(\text { mean } \pm \text { SD) }\end{array}$ & $0.03 \pm 0.16$ & $0 \pm 0$ & 0.324 \\
\hline \multicolumn{4}{|l|}{ Maternal alcohol biomarkers at V2: } \\
\hline GGT (>40 U/I): $n(\%)$ & $4(10.3)$ & $0(0.0)$ & 0.028 \\
\hline PEth ( $\geq 8 \mathrm{ng} / \mathrm{ml}): n$ (\%) & $3(7.7)$ & $0(0.0)$ & 0.070 \\
\hline UEtG ( $\geq 38 \mathrm{ng} / \mathrm{ml}): n(\%)$ & $3(7.7)$ & $0(0.0)$ & 0.070 \\
\hline UEtS ( $\geq 7.2 \mathrm{ng} / \mathrm{ml}): n(\%)$ & $9(23.1)$ & $0(0.0)$ & $<0.001$ \\
\hline$\% d C D T>2: n(\%)$ & $3(7.7)$ & $0(0.0)$ & 0.070 \\
\hline PEth-DBS ( $\geq 25 \mathrm{ng} / \mathrm{ml}$ ) & $7(18.0)$ & $0(0.0)$ & 0.002 \\
\hline
\end{tabular}

Cumulative alcohol use across pregnancy and periconceptional period

$\begin{array}{lccc}\text { AA/day (mean } \pm \text { SD) } & 0.44 \pm 0.72 & 0.001 \pm 0.004 & <0.001 \\ \text { AA/drinking day (mean } \pm \text { SD) } & 3.10 \pm 2.12 & 0.86 \pm 0.42^{\mathrm{a}} & <0.001 \\ \text { Positive for } \geq 1 \text { biomarker } & 21(53.9) & 0(0.0)) & <0.001\end{array}$

(V1 or V2) $n(\%)$

\section{SUBSTANCE USE ${ }^{\text {b: }}$}

\begin{tabular}{lccc} 
MAT (methadone, buprenorphine): & $22(56.4)$ & $19(35.2)$ & 0.057 \\
$\begin{array}{l}\text { Other opioids } \\
\text { (heroin or Rx opioids }{ }^{c} \text { ): }\end{array}$ & $14(35.9)$ & $11(20.4)$ & 0.105 \\
Marijuana: & $14(35.9)$ & $6(11.1)$ & 0.005 \\
Any tobacco use: & $21(53.9)$ & $15(27.8)$ & 0.017 \\
\hline
\end{tabular}

AA, absolute ounces of alcohol (1 standard drink equals $\sim 0.5 \mathrm{AA}$ ); \%dCDT, disialo carbohydrate deficient transferrin; DBS, dried blood spot; GGT, gamma-glutamyltranspeptidase; MAT, medication assisted therapy; PEth, phosphatidylethanol; UEtG, urine ethyl glucuronide; UEtS, urine ethyl sulfate.

a Sample size is limited to 5 controls who reported light drinking around LMP.

Either self-reported anytime in pregnancy or a positive urine drug panel at V1 or V2.

¿Used either as prescribed or recreational use.
Table 4. Infant developmental and behavioral outcomes by study group $(N=93)$

\begin{tabular}{|c|c|c|c|}
\hline Infant outcomes & $\operatorname{PAE}(n=39)$ & No-PAE $(n=54)$ & \\
\hline & Mean \pm SD & Mean \pm SD & $P$ \\
\hline BSID-III: Cognitive & $102.4 \pm 7.5$ & $101.3 \pm 9.3$ & 0.558 \\
\hline BSID-III: Language & $101.1 \pm 6.7$ & $99.7 \pm 7.1$ & 0.337 \\
\hline BSID-III: Motor & $97.0 \pm 11.2$ & $96.4 \pm 11.9$ & 0.821 \\
\hline \multicolumn{4}{|c|}{ Parenting Stress Index } \\
\hline $\begin{array}{l}\text { Parent-child dysfunctional } \\
\text { interaction } \%\end{array}$ & $36.6 \pm 28.1$ & $24.0 \pm 19.7$ & 0.019 \\
\hline Parental distress \% & $37.8 \pm 26.6$ & $31.3 \pm 30.1$ & 0.285 \\
\hline Difficult child \% & $30.1 \pm 25.9$ & $17.6 \pm 17.7$ & 0.011 \\
\hline Total stress \% & $32.5 \pm 26.2$ & $19.6 \pm 19.1$ & 0.011 \\
\hline \multicolumn{4}{|c|}{ Infant Behavior Questionnaire } \\
\hline Surgency & $65.9 \pm 10.4$ & $61.7 \pm 11.7$ & 0.081 \\
\hline Negative affect & $50.8 \pm 11.9$ & $43.5 \pm 13.9$ & 0.011 \\
\hline Effortful control & $65.9 \pm 8.6$ & $65.1 \pm 9.9$ & 0.717 \\
\hline \multicolumn{4}{|c|}{ Infant Sensory Profile } \\
\hline Low registration performance & n (\%) & n (\%) & 0.184 \\
\hline Less than others & $5(13.5)$ & $16(30.8)$ & \\
\hline Typical performance & $22(59.5)$ & $25(48.1)$ & \\
\hline More than others & $10(27.0)$ & $11(21.2)$ & \\
\hline Sensation seeking performance & & & 0.826 \\
\hline Less than others & $0(0)$ & $0(0)$ & \\
\hline Typical performance & $24(64.9)$ & $32(61.5)$ & \\
\hline More than others & $13(35.1)$ & $20(38.5)$ & \\
\hline Sensory sensitivity performance & & & 0.168 \\
\hline Less than others & $2(5.4)$ & $2(3.9)$ & \\
\hline Typical performance & $21(56.8)$ & $39(75.0)$ & \\
\hline More than others & $14(37.8)$ & $11(21.2)$ & \\
\hline $\begin{array}{l}\text { Sensation avoiding } \\
\text { performance }\end{array}$ & & & 0.436 \\
\hline Less than others & $4(10.8)$ & $2(3.9)$ & \\
\hline Typical performance & $25(67.6)$ & $40(76.9)$ & \\
\hline More than others & $8(21.6)$ & $10(19.2)$ & \\
\hline
\end{tabular}

BSID, Bayley Scales of Infant Development-III.

adjustment for infant sex, birth weight, single vs. two-parent household, maternal BDI, PSS, education, age, MAT, marijuana, and tobacco use (Table 5). In addition, trends were observed for an association between PAE and BSID Cognitive scale $(\hat{\beta}=3.44)$, PSI parent-child dysfunctional interaction $(\hat{\beta}=9.64)$, and IBQ surgency subscale $(\hat{\beta}=4.91)$; all $P$-values $<0.1$. There was also a trend for an association between PAE and a pattern of atypical sensory sensitivity (odds ratio $=2.29 ; 95 \%$ confidence interval: 0.93 ; 5.64 ) in the unadjusted model; however, results became nonsignificant after adjusting for covariates. Finally, no significant interactions were observed between PAE and infant sex with respect to any of the evaluated outcomes (all $P$-values $>0.05$; results not shown). 


\section{Behavioral outcomes in children with PAE Articles}

Table 5. Effect of PAE on infant developmental and behavioral outcomes

\begin{tabular}{|c|c|c|c|}
\hline Infant outcomes & $\hat{\beta}$ (unadjusted) & $\hat{\beta}\left(\right.$ adjusted $\left.^{\mathrm{a}}\right)$ & $\begin{array}{l}\eta_{p}^{2} \text { Detectable } \\
\text { effect size }\end{array}$ \\
\hline BSID-III: Cognitive & 1.06 & $3.44^{\#}$ & 0.0371 \\
\hline BSID-III: Language & 1.41 & 1.69 & 0.0132 \\
\hline BSID-III: Motor & 0.56 & 4.22 & 0.0306 \\
\hline \multicolumn{4}{|l|}{ Parenting Stress Index: } \\
\hline $\begin{array}{l}\text { Parent-child } \\
\text { dysfunctional } \\
\text { interaction \% }\end{array}$ & $12.7^{*}$ & $9.64^{\#}$ & 0.0418 \\
\hline Parental distress $\%$ & 6.49 & 7.52 & 0.0153 \\
\hline Difficult child \% & $12.5^{* *}$ & $13.9^{*}$ & 0.0752 \\
\hline Total stress $\%$ & $12.9^{* *}$ & $13.9^{*}$ & 0.0822 \\
\hline \multicolumn{4}{|c|}{ Infant Behavior Questionnaire: } \\
\hline Surgency & $4.23 \#$ & $4.91^{\#}$ & 0.0438 \\
\hline Negative affect & $7.33^{*}$ & $8.60^{* *}$ & 0.0935 \\
\hline Effortful control & 0.73 & -0.23 & 0.0001 \\
\hline Infant Sensory Profile & OR $(95 \% \mathrm{Cl})$ & & \\
\hline $\begin{array}{l}\text { Low registration } \\
\text { (atypical vs. typical) }\end{array}$ & $\begin{array}{c}0.63 \\
(0.27,1.48)\end{array}$ & $\begin{array}{c}0.62 \\
(0.20,1.91)\end{array}$ & $-{ }^{b}$ \\
\hline $\begin{array}{l}\text { Sensation seeking } \\
\text { (atypical vs. typical) }\end{array}$ & $\begin{array}{c}0.87 \\
(0.36,2.08)\end{array}$ & $\begin{array}{c}0.54 \\
(0.16,1.80)\end{array}$ & - \\
\hline $\begin{array}{l}\text { Sensory sensitivity } \\
\text { (atypical vs. typical) }\end{array}$ & $\begin{array}{c}2.29^{\#} \\
(0.93,5.64)\end{array}$ & $\begin{array}{c}2.10 \\
(0.69,6.40)\end{array}$ & - \\
\hline $\begin{array}{l}\text { Sensation avoiding } \\
\text { (atypical vs. typical) }\end{array}$ & $\begin{array}{c}1.60 \\
(0.62,4.11)\end{array}$ & $\begin{array}{c}2.06 \\
(0.63,6.76)\end{array}$ & - \\
\hline
\end{tabular}

$\hat{\beta}$ is the coefficient of the PAE variable.

adjusted for infant sex, birth weight, single vs. two-parent household, Beck Depression Inventory, maternal education, maternal age, MAT, marijuana, tobacco use, and Perceived Stress Scale score during pregnancy.

${ }^{b}$ The effect size measure for the logistic regression is odds ratio, which are presented in other columns of the table.

${ }^{*} P<0.01 ;{ }^{*} P<0.05 ;{ }^{\#} P<0.10$

\section{DISCUSSION}

Our results demonstrate that while global neurodevelopmental tests, such as BSID, might not yet identify deficits associated with PAE at 6 months of age, particularly moderate/light PAE, caregiver-reported assessments may provide an under-recognized opportunity to identify neurodevelopmental and behavioral concerns for infants with PAE. Temperament and behavioral characteristics, as well as sensory sensitivity of 6-month-old infants with PAE may be measurably altered. One of the striking findings earlier described by Streissguth et al. is that children with FAS and "fetal alcohol effects" have better long-term outcomes when identification/diagnosis is obtained at a young age (21). This finding has largely been attributed to the ability to begin subsequent interventions early that then led to better longterm outcomes. Therefore, additional measures that assess infant behavior and sensory processing may improve our ability to identify infants with PAE who may benefit from early intervention.

The 2016 Hoyme updated clinical guidelines for diagnosing FASD outline the following three critical domains to be assessed in children at-risk: (1) global intellectual ability; (2) behavior and self-regulation; and (3) adaptive skills (22). Our study indicates that measures of impaired emotional regulation (e.g., negative affect, "difficult child" rating) might be evident in 6-month-old children before deficits in other domains become apparent. Similar to our findings of increased negativity associated with PAE, two other studies reported increased stress reactivity and negative affect in infants with PAE seen at 5-7 months of age $(14,23)$. A longitudinal study by Molteno et al. (10) found that 13month-old infants with FAS had greater emotional withdrawal and decreased activity, while emotional withdrawal was also a significant predictor of a reduced IQ score at 9 years of age. These studies support our findings of emotional regulation difficulty and specifically ratings of higher negativity for those children with alcohol and drug exposure.

Children exposed to alcohol are also at higher risk for attachment problems and altered mother-infant interactions. Impairments in caregiver-child interaction and higher level of parental stress in families affected by PAE, found in our study, are concerning because the first year of life is an important period for maternal-infant bonding. Prior research has demonstrated that parental bonding and parental stress impact cognitive and behavioral outcomes in children, including executive functioning (24). In a recent review, Parolin and Simonelli (25) described the association between maternal substance abuse, which is often associated with dysfunctional caregiving environments, and attachment problems for the child. Newcomb and Locke found that substance use affected the parents' likelihood to show warmth, read their infant's cues, and predicted a style of parenting that included rejecting the child (26). It is, therefore, not surprising that we found the infants in this study were rated by their primary caregivers to have higher scores on parent-child dysfunctional interaction. Caregiver's rating of their infant as "difficult" on the PSI may be an indicator of parenting difficulty specifically related to the mother-child interaction. Emerging research indicates that caregivers raising children with FASD experience a high level of stress and well-being concerns, especially low-income families, those who care for adolescents (27), and have limited family resources (28). While research in this field is in formative stages, recent studies indicate that targeted family intervention programs (29) and parent training (30) might alleviate caregiver distress.

Our finding that the BSID-III only marginally sensitive to group differences was not surprising due to the limited sensitivity and predictability of the scale at this age; however, the study might have been under-powered to detect less than 10-point differences in the BSID. Earlier versions of the BSID were used in several prospective cohort samples of US infants with PAE during the 1980s and 1990s. While one of these 


\section{Articles | Bakhireva et al.}

studies found lower mental and motor BSID-II scores among 8-month-olds with $\mathrm{PAE}$, the magnitude of decrement in the PAE group compared to controls was only slight (31). Other similar studies found no significant group differences with the BSID-II among PAE vs. control infants $<1$ year of age, and mixed results in later follow-up of the infants between 12 and 24 months of age (32-35). While some more recent studies have identified neurodevelopmental deficits in infants with PAE during the first year of life using the BSID-II (12), there is increasing agreement that other measures might be more sensitive and specific $(13,14,36)$.

While we are not aware of any studies that have explored the ability to detect sensory processing deficits in a PAE population during the first year of life, limited existing data suggest that children with FASD who are of preschool age or greater may have significant abnormalities in multiple domains of sensory processing, and that these deficits may be related to other problem behaviors commonly observed with FASD (37-39). In a study of children 5-10 years with FASD, Franklin et al. (37) found that parents reported that up to $84 \%$ of the children with a diagnosis of FASD had both problem behaviors and sensory-processing impairments. A high correlation was also found between parents report on Dunn's Sensory Profile and the Child Behavior Checklist (CBCL). This supports the importance of including these types of parent report measures in studies of early identification of sensory problems that are associated with later problem behaviors. Animal studies show a consistent delay in auditory processing in animals with PAE suggesting that impaired myelination may play a role (40). Other studies indicate that somatosensory processing is also affected with evidence that the somatosensory cortex has reduced cortical representation in rats-an $11 \%$ reduction in forepaw representation (41). Xie et al. (42) extended these findings to low-exposure groups by demonstrating a similar pattern of reduced forepaw representations in somatosensory cortex. This is also consistent with our prior studies indicating delays in auditory (43) and visual (44) processing. The pattern of either sensory under- or over-sensitivity is consistent with other neurodevelopmental disorders, such as autism spectrum disorder, in which sensory over-/under- sensitivity is now considered one of the diagnostic criteria in the DSM-5. While it is unclear what underlying mechanisms lead to sensory sensitivity, these prior studies provide evidence for sensory processing deficits in human and animals studies of PAE.

This study has a number of strengths relative to prior studies on PAE. One of the primary strengths was the prospective cohort design in which mothers were assessed for alcohol consumption while pregnant. The same infants were followed to 6 months of age providing documented evidence of PAE rather than depending on retrospective self-report. This cohort also included infants with co-exposures, which is important since growing research acknowledges that polysubstance use in pregnancy is prevalent in the United States (45), and poses additional risks and challenges for the maternal-child pair. An additional strength was the inclusion of women on MAT, which served the purpose of controlling for environmental confounds such as socioeconomic status. As shown in Table 2, the PAE and No-PAE groups were well matched on educational level, family income, total SES score, and BDI score, which have previously been shown to impact infant development.

The results reported here are relevant to women who report moderate-to-low levels of alcohol consumption. In the DSM-5, a new diagnostic category, Neurodevelopmental Disorder Associated with PAE (ND-PAE), was introduced under "Conditions for further study," defining the PAE risk threshold as $>13$ drinks/month during pregnancy, but calling for prospective longitudinal research on the effects of this exposure level (18). Thus, our study on early physiological and behavioral indices associated with this level of exposure is addressing this call. Another strength of the ENRICH cohort is a state-of-the-art assessment of PAE with prospective repeated TLFB interviews during pregnancy and a comprehensive battery of ethanol biomarkers assessed twice (15).

This study is not without limitations with one of the main limitations being the relatively small sample size. The prospective design and the additional assessment of functional brain development (to be reported separately) limited the scope of the study to a well-characterized longitudinal sample as opposed to a larger cross-sectional sample. Despite this moderate sample size, differences in behavioral measures were identified; however, additional studies will be needed to replicate the reported findings. Second, this cohort includes individuals with other co-exposures. The individuals with MAT were included as a part of the initial study design to control for socioeconomic factors and were represented in both the control and the PAE groups. Within the MAT patient groups, there were co-exposures with other substances, as expected, but patients who consumed stimulants were excluded based on the known neurological effects. To account for these co-exposures, we adjusted for tobacco and marijuana consumption in multivariable modeling. Third, we recognize that caregiverreported outcomes might be more prone to recall bias relative to investigator-administered assessments, such as BSID and functional neuroimaging outcomes. However, given lowmoderate levels of exposure in the cohort we consider it highly unlikely that knowledge about prior PAE resulted in differential reporting of infant behavior.

Finally, we recognize that other pre- and postnatal factors not specifically evaluated in this study, e.g., early childhood adversity, postnatal maternal substance use, are important predictors of infant behavioral outcomes and parent-child attunement. However, in this sample there were only two children with protective services involvement. The distribution of key environmental factors (maternal education, family income, maternal BDI, involvement in early intervention programs) was similar between the groups. In addition, in a subset of 53 subjects who have completed Visit 4 (20-month evaluation), no differences were observed in the number of places child lived since birth (1.8 \pm 0.6 in PAE vs. $1.6 \pm 0.6$ in No-PAE groups). The effect of socio-environmental factors is minimized in our 


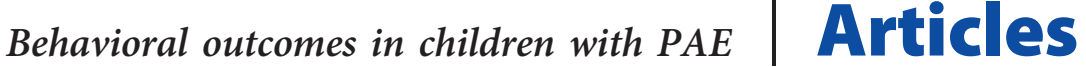

study since the PAE and No-PAE groups had similar SES background and substance use by design.

Based on the known limitations and the results of the current study, we envision important future directions. First, it is important to understand the longer-term effects of PAE, and the ENRICH study is designed to follow the children to 20 months of age to assess early markers of NB delays in PAE children. Data collection is ongoing and further results will be presented in future publications. While results presented in this study were adjusted for birth weight (which was highly collinear with gestational age of delivery) and the age of neurodevelopmental assessment was adjusted for prematurity, effect of birth weight and gestational age at delivery on ND outcomes should be carefully examined in larger future studies. The limited sample size also restricted the analysis that could be performed with the current data set examining different patterns of exposure throughout pregnancy, including timing, quantity, and frequency of drinking, as well as sex differences with respect to NB outcomes.

In summary, we believe this is the first study showing group differences in behavioral problems as early as 6 months of age in children with moderate prenatal alcohol exposure. The current results indicate that parental questionnaires may be able to provide an indication of PAE and may also point to potential areas as a target for early intervention. In particular, parents of children with PAE reported higher scores on the PSI, suggesting that family counseling and other supportive family-centered interventions may be beneficial, even at this early age.

\section{ACKNOWLEDGMENTS}

We are thankful to Crystal Aragon, Joy Van Meter, Steven Bishop, Sonnie Williams, Alyssa Ortega, Danielle Kabella, Lucinda Flynn, Rebecca Rieger, Natalia Moss, PhDc, and Shikhar Shrestha, $\mathrm{PhDc}$ for recruitment of study subjects, data collection, assistance with the data management and analysis. We are also in debt to William Rayburn, MD, MBA and Lawrence Leeman, MD, MPH for assisting with the research design and providing access to the study population.

\section{STATEMENT OF FINANCIAL SUPPORT:}

This work has been supported by the research grant from NIAAA/NIH (1R01AA021771). For statistical support, we acknowledge NIH support for the University of New Mexico Clinical \& Translational Science Center (UL1TR001449) and the Mountain West Clinical Translational Research Infrastructure Network (5 U54 GM104944).

Disclosure: The authors declare no conflict of interest.

\section{REFERENCES}

1. Substance Abuse and Mental Health Services Administration. Data Spotlight: The NSDUH Report [online], 2013 http://www.samhsa.gov/ data/sites/default/files/spot123-pregnancy-alcohol-2013/spot123-pregnancyalcohol-2013.pdf) (Accessed 28 Nov 2016).

2. May PA, Baete A, Russo J, et al. Prevalence and characteristics of fetal alcohol spectrum disorders. Pediatrics 2014;134:855-66.

3. Chasnoff IJ, Wells AM, King L. Misdiagnosis and missed diagnoses in foster and adopted children with prenatal alcohol exposure. Pediatrics 2015;135: 264-70.

4. Lange S, Rehm J, Anagnostou E, et al. Prevalence of externalizing disorders and autism spectrum disorder among children with fetal alcohol spectrum disorder: systematic review and meta-analysis. Biochem Cell Biol 2017: 1-11.
5. Coriale G, Fiorentino D, Di Lauro F, et al. Fetal alcohol spectrum disorder (FASD): neurobehavioral profile, indications for diagnosis and treatment. Riv Psichiatr 2013;48:359-69.

6. Mattson SN, Crocker N, Nguyen TT. Fetal alcohol spectrum disorders: neuropsychological and behavioral features. Neuropsychol Rev 2011;21:81-101.

7. Breiner P, Nulman I, Koren G. Identifying the neurobehavioral phenotype of fetal alcohol spectrum disorder in young children. J Popul Ther Clin Pharmacol 2013;20:e334-9.

8. Fuglestad AJ, Whitley ML, Carlson SM, et al. Executive functioning deficits in preschool children with fetal alcohol spectrum disorders. Child Neuropsychol 2015;21:716-31.

9. Molteno CD, Jacobson JL, Carter RC, et al. Infant symbolic play as an early indicator of fetal alcohol-related deficit. Infancy 2010;15:586-607.

10. Molteno CD, Jacobson JL, Carter RC, et al. Infant emotional withdrawal: a precursor of affective and cognitive disturbance in fetal alcohol spectrum disorders. Alcohol Clin Exp Res 2014;38:479-88.

11. Davies L, Dunn M, Chersich M, et al. Developmental delay of infants and young children with and without fetal alcohol spectrum disorder in the Northern Cape Province, South Africa. Afr J Psychiatry 2011;14:298-305.

12. Coles CD, Kable JA, Keen CL, et al. Dose and timing of prenatal alcohol exposure and maternal nutritional supplements: developmental effects on 6-month-old infants. Matern Child Health J 2015;19:2605-14.

13. Mesa DA, Kable JA, Coles CD, et al. The use of cardiac orienting responses as an early and scalable biomarker of alcohol-related neurodevelopmental impairment. Alcohol Clin Exp Res 2017;41:128-38.

14. Haley DW, Handmaker NS, Lowe J. Infant stress reactivity and prenatal alcohol exposure. Alcohol Clin Exp Res 2006;30:2055-64.

15. Bakhireva LN, Lowe JR, Gutierrez HL, et al. Ethanol, neurodevelopment, infant and child health (ENRICH) prospective cohort: study design considerations. Adv Pediatr Res 2015;2:1-13.

16. Jacobson SW, Chiodo LM, Sokol RJ, et al. Validity of maternal report of prenatal alcohol, cocaine, and smoking in relation to neurobehavioral outcome. Pediatrics 2002;109:815-25.

17. Bakhireva LN, Cano S, Rayburn WF, et al. Advanced gestational age increases serum carbohydrate-deficient transferrin levels in abstinent pregnant women. Alcohol Alcohol 2012;47:683-7.

18. Kable JA, Mukherjee RA. Neurodevelopmental disorder associated with prenatal exposure to alcohol (ND-PAE): a proposed diagnostic method of capturing the neurocognitive phenotype of FASD. Eur J Med Genet 2017;60:49-54.

19. Cohen S, Kamarck T, Mermelstein R. A global measure of perceived stress. Journal of Health and Social Behavior. 1983;24:385-96.

20. Flak AL, Su S, Bertrand J, et al. The association of mild, moderate, and binge prenatal alcohol exposure and child neuropsychological outcomes: a meta-analysis. Alcohol Clin Exp Res 2014;38:214-26.

21. Streissguth AP, Bookstein FL, Barr HM, et al. Risk factors for adverse life outcomes in fetal alcohol syndrome and fetal alcohol effects. J Dev Behav Pediatr 2004;25:228-38.

22. Hoyme HE, Kalberg WO, Elliott AJ, et al. Updated clinical guidelines for diagnosing fetal alcohol spectrum disorders. Pediatrics 2016;138:e20154256.

23. Lowe J, Handmaker N, Aragon C. Impact of mother interactive style on infant affect among babies exposed to alcohol in utero. Infant Ment Health J 2006;27: 371-82.

24. de Cock ESA, Henrichs J, Klimstra TA, et al. Longitudinal associations between parental bonding, parenting stress, and executive functioning in toddlerhood. J Child Fam Stud 2017;26:1723-33.

25. Parolin M, Simonelli A. Attachment theory and maternal drug addiction: the contribution to parenting interventions. Front Psychiatry 2016;7:152.

26. Newcomb MD, Locke TF. Childhood adversity and poor mothering: consequences of polydrug abuse use as a moderator. Addict Behav 2005;30: $1061-4$.

27. Bobbitt SA, Baugh LA, Andrew GH, et al. Caregiver needs and stress in caring for individuals with fetal alcohol spectrum disorder. Res Dev Disabil 2016;55:100-13.

28. Paley B, O'Connor M J, Frankel F, et al. Predictors of stress in parents of children with fetal alcohol spectrum disorders. J Dev Behav Pediatr 2006;27:396-404. 


\section{Articles | Bakhireva et al.}

29. Leenaars LS, Denys K, Henneveld D, et al. The impact of fetal alcohol spectrum disorders on families: evaluation of a family intervention program. Community Ment Health J 2012;48:431-5.

30. Turner K, Reynolds JN, McGrath P, et al. Guided internet-based parent training for challenging behavior in children with fetal alcohol spectrum disorder (strongest families FASD): study protocol for a randomized controlled trial. JMIR Res Protoc 2015;4:e112.

31. Streissguth AP, Martin DC, Martin JC, et al. The Seattle longitudinal prospective study on alcohol and pregnancy. Neurobehav Toxicol Teratol 1981;3:223-33.

32. Fried PA, Watkinson B. 12- and 24-month neurobehavioural follow-up of children prenatally exposed to marihuana, cigarettes and alcohol. Neurotoxicol Teratol 1988;10:305-13.

33. Richardson GA, Day NL, Goldschmidt L. Prenatal alcohol, marijuana, and tobacco use: infant mental and motor development. Neurotoxicol Teratol 1995;17:479-87.

34. Coles CD, Smith IE, Falek A. Prenatal alcohol exposure and infant behavior: immediate effects and implications for later development. Adv Alcohol Subst Abuse 1987;6:87-104.

35. Jacobson JL, Jacobson SW, Sokol RJ, et al. Teratogenic effects of alcohol on infant development. Alcohol Clin Exp Res 1993;17:174-83.

36. Oberlander TF, Jacobson SW, Weinberg J, et al. Prenatal alcohol exposure alters biobehavioral reactivity to pain in newborns. Alcohol Clin Exp Res 2010;34:681-92.

37. Franklin L, Deitz J, Jirikowic T, et al. Children with fetal alcohol spectrum disorders: problem behaviors and sensory processing. Am J Occup Ther 2008;62:265-73.
38. Jirikowic T, Olson HC, Kartin D. Sensory processing, school performance, and adaptive behavior of young school-age children with fetal alcohol spectrum disorders. Phys Occup Ther Pediatr 2008;28: 117-36.

39. Wengel T, Hanlon-Dearman AC, Fjeldsted B. Sleep and sensory characteristics in young children with fetal alcohol spectrum disorder. J Dev Behav Pediatr 2011;32:384-92.

40. Church MW, Abel EL, Kaltenbach JA, et al. Effects of prenatal alcohol exposure and aging on auditory function in the rat: preliminary results. Alcohol Clin Exp Res 1996;20:172-9.

41. Margret CP, Chappell TD, Li CX, et al. Prenatal alcohol exposure (PAE) reduces the size of the forepaw representation in forepaw barrel subfield (FBS) cortex in neonatal rats: relationship between periphery and central representation. Exp Brain Res 2006;172:387-96.

42. Xie N, Yang Q, Chappell TD, et al. Prenatal alcohol exposure reduces the size of the forelimb representation in motor cortex in rat: an intracortical microstimulation (ICMS) mapping study. Alcohol 2010;44: 185-94.

43. Stephen JM, Kodituwakku PW, Kodituwakku EL, et al. Delays in auditory processing identified in preschool children with FASD. Alcohol Clin Exp Res 2012;36:1720-7.

44. Coffman BA, Kodituwakku P, Kodituwakku EL, et al. Primary visual response (M100) delays in adolescents with FASD as measured with MEG. Hum Brain Mapp 2013;34:2852-62.

45. Jarlenski M, Barry CL, Gollust S, et al. Polysubstance use among US women of reproductive age who use opioids for nonmedical reasons. Am J Public Health 2017;107:1308-10. 